\title{
Minimally invasive transatrial mitral valve replacement in mitral annular calcification
}

\author{
Alexander P. Nissen ${ }^{1,2}$, Joseph Lamelas ${ }^{3}$, Isaac George ${ }^{4}$, Juan Umana-Pizano $^{1}$, Tom C. Nguyen ${ }^{1}$ \\ ${ }^{1}$ Department of Cardiothoracic and Vascular Surgery, University of Texas Health Science Center Houston, McGovern Medical School, Houston, \\ TX, USA; ${ }^{2}$ Department of Surgery, San Antonio Military Medical Center, Fort Sam Houston, TX, USA; ${ }^{3}$ Division of Cardiothoracic Surgery, \\ Michael E. DeBakey Department of Surgery, Baylor College of Medicine and Texas Heart Institute at CHI Baylor St. Luke's Medical Center, \\ Houston, TX, USA; ${ }^{4}$ Division of Cardiothoracic Surgery, New York Presbyterian Hospital—College of Physicians and Surgeons of Columbia \\ University, New York, NY, USA \\ Correspondence to: Tom C. Nguyen, MD, FACS, FACC. Director of Minimally Invasive Valve Surgery, Associate Professor of Cardiothoracic Surgery, \\ 6400 Fannin St, Suite 2850, Houston, TX, USA. Email: tom.c.nguyen@gmail.com.
}

Submitted Sep 14, 2018. Accepted for publication Oct 09, 2018.

doi: 10.21037/acs.2018.10.07

View this article at: http://dx.doi.org/10.21037/acs.2018.10.07

\section{Introduction}

Mitral annular calcification (MAC) is associated with increased cardiovascular morbidity and mortality in patients undergoing mitral valve replacement (1). In high-risk patients, an alternative to conventional surgical techniques involves placement of a balloon-expandable transcatheter valve, either through a transapical, transseptal (TS), or open surgical approach $(2,3)$. While TS delivery is an attractive option, up to $75 \%$ of patients may not be candidates for TS implantation based on non-circumferential MAC (see Figure 1), an elliptical-shaped annulus or annulus too large to seal the transcatheter valve with subsequent risk for either paravalvular leak (PVL) or valve embolization (2). Other exclusion criteria include a long anterior mitral leaflet, or highly calcified subvalvular apparatus, which risk left ventricular outflow tract obstruction (LVOTO), the presence of left atrial or ventricular thrombus, which risks of stroke during transcatheter manipulation, or the need for concomitant cardiac operations. Among patients treated with transcatheter valve in MAC via an open approach, there is sparse literature detailing a minimally invasive approach, which represents an attractive option in this high-risk cohort (4).

\section{Clinical vignette}

An 84-year-old woman with severe aortic stenosis, severe mitral stenosis, persistent atrial fibrillation and pulmonary hypertension, complained of worsening shortness of breath and lower extremity edema. Preoperative transesophageal echocardiography (TEE) demonstrated an aortic valve area of $0.62 \mathrm{~cm}^{2}$, mean aortic gradient of $48 \mathrm{mmHg}$, mean mitral gradient of $19 \mathrm{mmHg}$, a discrete patent foramen ovale (PFO) and moderate tricuspid regurgitation. Preoperative computed tomography (CT) demonstrated severe MAC extending into the left ventricle (see Figure 1). Her calculated STS risk score for isolated mitral replacement was $9.8 \%$ and STS risk for isolated aortic valve replacement was $7.4 \%$. The STS risk calculator does not allow computation of mortality risk for simultaneous AVR and MVR and in the setting of severe noncircumferential MAC, our patient was deemed very high-risk for TS mitral valve replacement in MAC. After discussion with our heart team, we planned on combined transcatheter aortic valve replacement (TAVR), minimally invasive mitral replacement with the Edwards Sapien ${ }^{\mathrm{TM}} \mathrm{S} 3$ valve in MAC and PFO closure.

\section{Surgical technique}

\section{Preparation}

Detailed review of all preoperative imaging is critical. $\mathrm{CT}$ is used for incision planning, outlining the MAC circumferentially for tentative valve sizing, planning annular suture placement, measurement of anterior leaflet length and thorough assessment of any troublesome areas within the MAC (caseous MAC, areas extending into the LV). The anterior leaflet and left ventricular outflow tract (LVOT) 

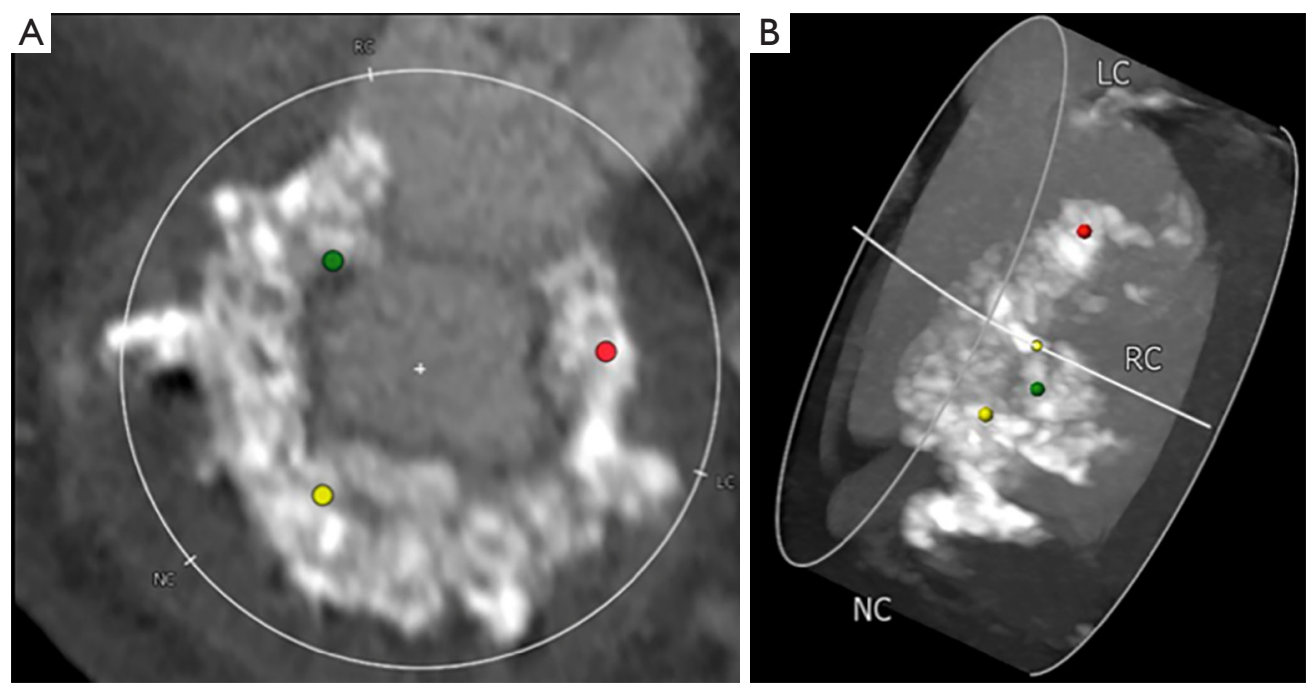

Figure 1 Representative orthogonal (A) and 3D reconstruction (B) images of severe noncircumferential MAC with left ventricular extension in a patient planned to undergo open transcatheter mitral replacement.

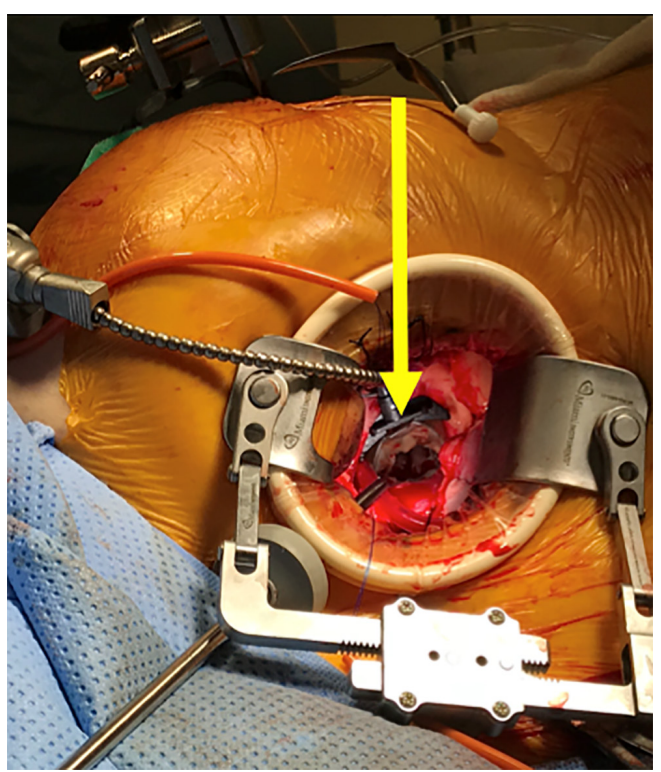

Figure 2 A representative image of mitral exposure through right minithoracotomy. Yellow arrow demonstrates significant distance from traditional sternotomy incision to mitral valve, compared to the direct en-face view through the right chest.

dimension should also be measured by echocardiography. If available, $3 \mathrm{Mensio}^{\mathrm{TM}}$ imaging can allow $3 \mathrm{D}$ visualization with the transcatheter valve superimposed to further assess risk of LVOTO. TEE or cardiac MRI are preferred over CT when deciding whether to perform septal myectomy. Despite these studies, a thorough intraoperative assessment remains crucial.

\section{Exposition}

The patient is positioned supine with the right arm above the head. After uneventful transfemoral TAVR, femoral venous and arterial cannulation are used for peripheral cardiopulmonary bypass (CPB). A $5 \mathrm{~cm}$ right minithoracotomy incision is made in the fourth interspace. The pleura is entered, and pericardium opened above the phrenic nerve. Appropriate pericardial suture placement provides effective diaphragmatic retraction and exposure. Cardioplegic arrest is facilitated by aortic cross-clamping, taking care to place the clamp as high as possible, which is key in TAVR patients to avoid injury to the valve frame. The left atrium is entered via Sondergaard's groove. A left atrial retractor inserted through a separate incision provides excellent exposure of the mitral valve (Figure 2). One must avoid aggressive retraction in patients with prior TAVR to prevent distortion of the TAVR valve (5).

\section{Operation}

The native mitral valve is inspected and the anterior leaflet resected to minimize risk of LVOTO, leaving a $2-3 \mathrm{~mm}$ rim of tissue. The posterior leaflet and MAC are not excised to minimize risk of atrioventricular disruption. We place as many pledgeted annular sutures as possible, such that knots are tied on the atrial side. Complete circumferential suture 
placement is not mandatory, but it is important to place sutures at each commissure, between P1-P2 and P2-P3, and any areas of deep indentation. Additional sutures may need to be placed in the inter-trigonal region for additional support if this area is devoid of calcium. If dense MAC prevents annular suture placement, anchoring in residual leaflet tissue should suffice. The optimal method for valve anchoring is still evolving, with nuances amongst techniques of the senior authors outlined in the accompanying video.

Valve sizing is performed with the Sapien ${ }^{\mathrm{TM}}$ delivery balloon, which is inflated until snug to determine appropriate valve size. The appropriately sized valve is prepared by sewing a $0.75-1 \mathrm{~cm}$ felt strip circumferentially with 5-0 polypropylene tied to itself to not constrain the valve when fully deployed. The commissures of the $\mathrm{S} 3$ valve are marked, to facilitate orientation and prevent LVOTO. The valve is crimped onto the delivery device, though full crimping is not mandatory, and asymmetrical crimping may occur.

The delivery system is positioned for valve deployment, with approximately $50-60 \%$ of the felt strip in a supraannular position. Again, the exact height of valve deployment is evolving, and erring towards more intra-atrial deployment may help avoid LVOTO. The valve is oriented by the lead surgeon, as coaxial as possible with the mitral annulus. A second operator, experienced with Sapien ${ }^{\mathrm{TM}}$ valve deployment, inflates the balloon very slowly, and until snug. Avoid overinflation to prevent rupture. The balloon is deflated and previous annular sutures passed through the felt skirt/valve and tied. The balloon may be reinserted at this point and inflated for 10-20 seconds for full expansion. The balloon is removed and the valve checked for leaks. Sites of PVL may be occluded with additional annular sutures or a small Amplatzer ${ }^{\mathrm{TM}}$ plug.

\section{Completion}

The left atriotomy is closed, the heart de-aired, and after cross-clamp removal, postprocedural TEE is critical to assess valve function, any PVL or LVOTO, and the function of the TAVR valve. We obtain a pre-discharge transthoracic echocardiogram on all patients and again at 3 months postoperatively.

\section{Comments}

For selected high-risk patients with MAC requiring mitral replacement, a minimally invasive approach to open transcatheter valve replacement represents a viable option. We highlighted the technique in our patient, including methods to minimize PVL and LVOTO, as well as methods for appropriate valve sizing and safe deployment, recognizing nuances in technique that are still evolving.

\section{Acknowledgements}

None.

\section{Footnote}

Conflicts of Interest: J Lamelas: Consultant of Edwards LifeSciences, Medtronic, St. Jude Medical; Miami Instruments-Partnership interest without compensation. I George: Consultant of Edwards LifeSciences, Medtronic. TC Nguyen: Consultant of Edwards LifeSciences, Abbott, LivaNova. Other authors have no conflicts of interest to declare.

\section{References}

1. Eleid MF, Foley TA, Said SM, et al. Severe Mitral Annular Calcification: Multimodality Imaging for Therapeutic Strategies and Interventions. JACC Cardiovasc Imaging 2016;9:1318-37.

2. Praz F, Khalique OK, Lee R, et al. Transatrial implantation of a transcatheter heart valve for severe mitral annular calcification. J Thorac Cardiovasc Surg 2018;156:132-42.

3. Guerrero M, Dvir D, Himbert D, et al. Transcatheter Mitral Valve Replacement in Native Mitral Valve Disease With Severe Mitral Annular Calcification: Results From the First Multicenter Global Registry. JACC Cardiovasc Interv 2016;9:1361-71.

4. Lamelas J, Silva GV, Chatterjee S. Minimally Invasive Direct Access Balloon-Expandable Transcatheter Mitral Valve Replacement for Extensive Mitral Annular Calcification after Transcatheter Aortic Valve Replacement. Innovations (Phila) 2018;13:222-5.

5. Nguyen TC, Umana-Pizano JB, Landinez GP, et al. Minimally Invasive SAPIEN in Mitral Annular Calcification Following Transcatheter Aortic Valve Replacement: Feasibility and Lessons Learned. Semin Thorac Cardiovasc Surg 2018;30:290-2.

Cite this article as: Nissen AP, Lamelas J, George I, Umana-Pizano J, Nguyen TC. Minimally invasive transatrial mitral valve replacement in mitral annular calcification. Ann Cardiothorac Surg 2018;7(6):827-829. doi: 10.21037/ acs.2018.10.07 